\title{
Inhibition of Fibrogenesis upon Hydralazine-induced DNA Demethylation
}

\section{Zeisberg $\mathrm{EM}^{1,2}$ and Zeisberg $\mathbf{M}^{2,3 *}$}

${ }^{1}$ Department of Cardiology and Pneumology, University Medical Center Göttingen, Georg-August University, Göttingen, Germany ${ }^{2}$ Center for Cardiovascular Research (DZHK), Robert Koch Street 40, Göttingen, Germany

${ }^{3}$ Department of Nephrology and Rheumatology, University Medical Center Göttingen, Georg August University, Göttingen, Germany

\begin{abstract}
Chronic progressive kidney disease (CKD) remains an unsolved problem in clinical Nephrology as neither biomarkers to predict progression towards end-stage renal failure in individual patients nor specific drugs to inhibit decline of kidney function are available in the clinic yet. In this regard, the prototypical epigenetic mechanism of $\mathrm{CpG}$ island promoter methylation has emerged as modulator of disease progression with utilities both as biomarker and as therapeutic target. Recent studies demonstrated the potential of hydralazine, a long-established antihypertensive drug with de-methylating activity, to reverse aberrant $\mathrm{CpG}$ island promoter aberration and ameliorate progression of chronic kidney disease. Here we review contribution of aberrant promoter methylation to progression of fibrogenesis and mechanisms underlying hydralazine's demethylating activity and discuss possible translational implications.
\end{abstract}

Keywords: Kidney; Fibrosis; Epigenetics; Methylation; Tet3; Fibroblast; Hydralazine

\section{Introduction}

While progression of chronic kidney disease remains an unsolved challenge, recent pre-clinical studies revealed that that the epigenetic mechanism of DNA-hypermethylation contributes to progression of fibrosis and that fibrogenesis in the kidney could be ameliorated through administration of the de-methylating compound hydralazine. Importantly, such de-methylating anti-fibrotic activity was achieved at doses which were substantially lower than standard anti-hypertensive regimen, which could be also confirmed by retrospective analysis of patients with nephrosclerosis. Because hydralazine has been in clinical use as anti-hypertensive drug for over 50 years, we here discuss an opportunity to address a pressing clinical problem by re-purposing of an old and safe drug.

\section{Contribution of renal fibrosis to progression of chronic kidney disease}

Renal fibrosis, also referred to as tubulointerstitial fibrosis (TIF) is of major importance for clinical nephrology as it is the common pathway leading to end-stage renal failure in patients [1,2]. Currently, there are no specific treatments available in the clinic to inhibit (or even reverse) progressive renal fibrosis [3,4]. Furthermore, there is a paucity of biomarkers available to predict the individual risk of patients with renal disease to develop end-stage renal failure [1]. Renal fibrosis - just like fibrosis in other organs - is a pathologic scarring process [5-7]. A hallmark of fibrosis is that - as opposed to physiologic wound healing - the active scarring does not cease once the initial insult has been contained, but it becomes an autonomous process [5].

Activated fibroblasts are commonly considered main mediators of renal fibrosis 5 (just like in any other organ) $[8,9]$. The term "activated" refers to the observation, that fibroblasts associated with wound healing or fibrosis display an increased proliferative activity and increased production of extracellular matrix (ECM) constituents and formation of stress fibers [5]. It is well established that activated fibroblasts isolated from fibrotic kidneys maintain their activated state when cultured in vitro, as primary "fibrotic" fibroblasts are characterized by an increased proliferation rate and increased secretion of ECM constituents [10]. It has been hypothesized that such maintenance of the activated stage reflects the failure of the fibroblasts in the fibrotic kidney to return to their resting state as they do in physiologic wound healing [10]. This phenomenon is not only observed in fibrotic kidney fibroblasts, but is also true for fibroblasts isolated from any given organ $[8,11]$. Furthermore, such pathological maintenance of the activated state has received increased attention in the cancer field in the case of "carcinoma-associated-fibroblasts" [12,13] (Figure 1).

\section{Epigenetic modifications}

Perpetuated disease progression of fibrosis in contrast to physiological wound repair points to a role of epigenetics in fibrogenesis [14-16]. Epigenetic modifications in principle can "stably alter gene expression without altering the primary nucleotide sequence" [17]. The two principal epigenetic mechanisms are histone modifications and DNA-methylation. In a simplified view, genes can be either "open" for transcription or "locked" [18]. "Open" genes that are accessible for polymerases are associated with un-methylated promoter regions and with histones, which are permissive for gene transcription ("activating histone marks"). "Locked" genes are associated with DNAhypermethylation of the promoter region and enrichment of histone modifications which prevent gene transcription ("inhibitory histone marks") [17]. While understanding of the role of histone modifications in progression of chronic kidney disease is still rudimentary, there substantial is evidence from past years which demonstrates a causal role of aberrant DNA methylation in renal fibrogenesis (and in fibrosis in other organs as well) [19-23]. DNA methylation (syn. promoter CpG island methylation) is a process in which methyl groups are added to clusters of cytosine nucleotides within promoter DNA regions. DNA methylation typically acts to repress gene transcription, whereas unmethylated promoters are open for transcription. DNA methylation plays important roles in physiologic processes including cell differentiation during development, genomic imprinting, $\mathrm{X}$-chromosome inactivation, and repression of repetitive elements

${ }^{*}$ Corresponding author: Prof. Dr. med. Michael Zeisberg, Department of Nephrology and Rheumatology, Göttingen University Medical Center, Georg August University, Robert Koch Str. 40, 37075 Göttingen, Germany, E-mail: mzeisberg@med.uni-goettingen.de

Received: January 11, 2016; Accepted: April 07, 2016; Published: April 19, 2016

Citation: Zeisberg EM, Zeisberg M (2016) Inhibition of Fibrogenesis upon Hydralazine-induced DNA Demethylation. J Kidney 2: 124. doi:10.4172/24721220.1000124

Copyright: (c) 2016 Zeisberg EM, et al. This is an open-access article distributed under the terms of the Creative Commons Attribution License, which permits unrestricted use, distribution, and reproduction in any medium, provided the original author and source are credited. 


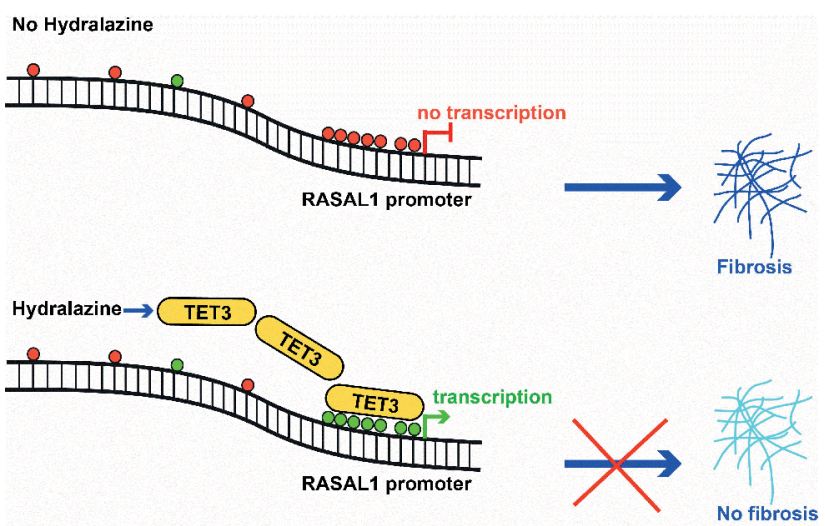

Figure 1: Schematic illustration of Hydralazine's de-methylating antifibrotic activity. Methylation of RASAL1 CpG promoter islands (red circles) causes transcriptional RASAL1 suppression and subsequently contributes to fibroblast accumulation (top picture). Hydralazine induces expression of the enzyme TET3 which via its CXXC binding motif targets CpG promoter islands of select genes (green circles), including RASAL1. TET3 facilitates hydroxymethylation of methylated cytosine residues, which subsequently induces

RASAL1 transcription, CpG island promoter de-methylation and inhibits fibrogenesis.

$[18,24]$. Suppression of gene transcription through DNA methylation in differentiating cells is normally permanent and prevents cells from converting into a different cell type [25]. DNA methylation suppresses gene transcription by either physically blocking the binding of transcription factors, or by recruiting methyl-CpG-binding domain proteins (MBDs), which then recruit additional chromatin remodeling proteins forming heterochromatin. In humans there are four known enzymes which can methylate DNA in principle, termed DNA methyl transferases Dnmt1, Dnmt3a, DNMt3b and DnmtL [18]. During development de novo methylation is carried out by Dnmt3a, Dnmt3b and Dnmt3L, whereas Dnmt1 mostly is necessary to preserve DNA methylation during DNA replication (otherwise DNA replication would result in unmethylated daughter strands) $[26,27]$.

\section{Contribution of aberrant DNA methylation to fibroblast activation and fibrosis}

Progression of chronic kidney disease is associated with substantial and consistent changes of renal methylation patters, providing correlative evidence for contribution to disease progression $[28,29]$. First causal evidence for a role of aberrant DNA methylation in kidney fibrosis stems from studies, in which fibrosis in mouse models of renal fibrosis were ameliorated by treatment with the de-methylating drug 5’Azacytidine (5’Aza) [19]. Evidence for direct causal contribution of aberrant DNA methylation was made possible by identification of genes which are selectively methylated in kidney fibrosis [19]. In this regard, our group in a genome-wide methylation screen, by comparing fibroblasts from fibrotic and non-fibrotic kidney biopsies, identified the gene RASAL1 (which encodes for Ras-Gap-like protein 1, RASAL1), an inhibitor of Ras signaling [19].

In general, the Ras superfamily of proteins is a family of structurally related small GTPase signal transduction molecules, with Ras being the prototypical member [30]. Ras stands for "Rat sarcoma", reflecting that Ras was originally discovered through studies of cancer causing viruses (HRas was discovered in Harvey sarcoma virus and KRas in Kirsten sarcoma virus). Ras is the most common oncogene (i.e., $90 \%$ of pancreatic cancers and 50\% of thyroid cancers are causally linked to activating Ras mutations) and as a consequence most knowledge on Ras signaling in health and disease stems from cancer research [30]. In humans three Ras genes (HRAS, NRAS, KRAS) encode four proteins $(\mathrm{H}-, \mathrm{N}$ - and $\mathrm{K}$-as $4 \mathrm{~A}$ and $\mathrm{K}$-ras4 $\mathrm{B}$ which are generated through alternative splicing) [31,32]. Ras signaling is complex, as multiple effector pathways can be activated - all with established contributions in progression of chronic kidney disease $[33,34]$. The most prominent Ras-signaling pathway is initiated by phosphorylation of Raf, which in turn phosphorylates mitogen activated protein kinases ERK1 and ERK2, which ultimately enhance cell cycle progression [30]. In addition, Ras interacts directly with the phosphatidylinositol-3-kinases (PI3-Ks), leading to activation of the kinase phosphinositide-dependent protein kinase-1 (PDK1) and Akt kinase 30. Akt has a strong anti-apoptotic effect by phosphorylating various targets and is considered to play a major role in the survival signal which is generated by Ras proteins [30]. Ras proteins are $\mathrm{G}$ proteins which can be most adequately described as on-off switches: They are inactive when GDP-bound and active when GTP bound. Under physiological conditions GDP-bound Ras proteins are anchored to the cell membrane. Upon growth factor stimulation, so called guanine nucleotide exchange factors (GEFs) facilitate conversion of inactive Ras-GDP to Ras-GTP. Well-known examples of such GEFs include Son of Sevenless and cdc25 [35,36]. Ras-GTP is inactivated through hydrolysis of GTP to GDP. This is facilitated through so-called GTPase activating proteins (Ras-GAPs) [35,36]. (The name refers to the fact that Ras has its own GTPase activity, which is however insufficient to hydrolyze GTP itself and requires an extrinsic catalytic activator - the Ras-GAPs.) RASAL1 is referred to as Ras-GAPlike protein, which catalyzes Ras-inactivation by binding to GTP-Ras and catalyzing hydrolysis to GDP-Ras [37]. This means that depletion of RASAL1 through CpG island promoter methylation results in intrinsic Ras hyperactivity [38]. RASAL1 depletion in cultured renal fibroblasts recapitulates important aspects of fibroblast activation: Increased proliferative activity, increased collagen production and stress fiber formation [19]. Furthermore, administration of a broad Ras-GTP inhibitor "FTS" ameliorated renal fibrosis in mice (which had been challenged with either folic acid or nephrotoxic serum nephritis) through blunting the increased intrinsic Ras-GTP activity, which is observed in kidneys undergoing fibrogenesis [19]. Overexpression of RASAL1 in transgenic mice ameliorates renal fibrosis, providing definitive evidence that transcriptional suppression of RASAL1 causally contributes to renal fibrogenesis.

\section{RASAL1 promoter CpG island methylation is a constituent of renal fibrosis irrespective of the underlying disease}

Experimental kidney fibrosis is associated with Rasal1 hypermethylation and decreased Rasall expression, including mouse models of folic acid-induced nephropathy, nephrotoxic serum nephritis [19], unilateral ureteral obstruction (UUO), diabetic nephropathy through administration of streptozotocin (DN) [39], COL4A3-deficient "Alport"-mice (COL4A3KO) and 5/6 nephrectomy (5/6 NX) [39]. In whole kidney biopsies from patient with varying degree of fibrosis and corresponding fibroblasts, severe fibrosis was associated with RASAL1 hypermethylation and transcriptional silencing of RASAL1 [39]. In this regard, transcriptome analysis data on larger cohorts reveal that chronic kidney disease due to minimal change nephropathy and hypertensive nephrosclerosis also correlated with decreased RASAL1 expression [39]. In summary, RASAL1 is consistently hypermethylated 
in kidney fibrosis irrespective of the underlying cause, suggesting that ensuing Ras-GTP hyperactivity might be a highly relevant pathway of renal fibrogenesis.

Consistency of RASAL1 methylation in fibrotic kidney biopsies not only corroborates contribution of RASAL1 hypermethylation to disease contribution, but also raises the possibility of RASAL1 methylation as biomarker of kidney fibrosis progression. However, the need for a renal biopsy to assess intrarenal degree of RASAL1 methylation is prohibitive in this regard. On the other hand, the degree of renal RASAL1 methylation is reflected by levels of circulating Rasal1 promoter DNA fragments in peripheral blood [40]. Such correlation of fibrosis, decreased renal RASAL1 expression and increased levels of methylated circulating RASAL1 DNA fragments can be found in mice challenged kidney fibrosis models [40]. Our group also explored the possibility that circulating methylated RASAL1 promoter fragments could similarly reflect intrarenal RASAL1 methylation (and possibly fibrosis) in patients with CKD as they do in mice all single-center cohort of patients with varying degree of renal fibrosis at time of biopsy, renal fibrosis was inversely correlated with intrarenal RASAL1 mRNA expression levels [40]. The degree of intrarenal RASAL1 promoter $\mathrm{CpG}$ island methylation correlated with the degree of renal fibrosis, irrespective of the underlying disease $[39,40]$. Furthermore, we observed that degrees of intrarenal RASAL1 promoter CpG island methylation correlated with levels of methylated RASAL1 promoter fragments [40]. Few patients displayed significantly increased levels of methylated RASAL1 promoter fragments at CKD stage 1, at CKD stage 4 all patients analyzed had increased levels of circulating RASAL1 DNA. Importantly, circulating RASAL1 promoter fragment levels correlated better with degree of interstitial fibrosis at the time of biopsy was superior to that of estimated glomerular filtration rate (eGFR), serum creatinine or blood urea nitrogen (BUN) levels [40]. Current limitations include the labor intensity of currently established assays which involves immunoprecipitation of methylated DNA from blood samples and the lack of large prospective clinical studies. In summary, methylation of RASAL1 contributes causally to progression of kidney fibrosis, is an attractive therapeutic target and there is promise of utility as biomarker for kidney fibrosis. Furthermore, with the possibility of therapeutic de-methylation, there comes the attractive possibility of a biomarker-stratified epigenetic therapy which could be monitored for therapeutic efficacy and adequate dosing.

\section{Mechanisms of hydralazine-mediated demethylation}

In general, methylated DNA can be de-methylated either through passive or active mechanisms. Passive de-methylation can occur when methylation marks are not copied on the newly synthesized strands meaning that during a first cell division, hemi-methylated DNA is generated and the methylation mark is entirely removed upon the second cell division [41]. For rapid de-methylation to occur, active de-methylation mechanisms involve oxidation of methylated cytosine $(5 \mathrm{mC})$ to generate 5-hydroxymethyl-cytosine (5hmC), 5-formylcytosine (5fC) and 5-carboxyl-cytosine ( $5 \mathrm{caC})$; subsequent base excision and replacement with naked cytosine are a consequence [41]. This pathway is recapitulated when kidney cells are exposed to hydralazine: Hydralazine induces expression of Ten-Eleven Translocation enzyme-3 (Tet3), which catalyzes hydroxymethylation of aberrantly methylated genes (such as Rasal1) [40]. Such hydroxymethylation (which itself is sufficient to increase transcriptional activity of previously methylated genes) is followed by carboxylation of $5 \mathrm{hm}$-cytosine residues [40]. In subsequent steps hydralazine induces replacement of hydroxymethylated cytosines with naked cytosine bases: First, hydroxymethylated cytosines are further oxidized to 5-formyl-cytosine $(5 \mathrm{fC})$ and then to 5-carboxyl-cytosine $(5 \mathrm{caC})$ before excision of $5 \mathrm{caC}$ by the DNA glycosylase Tdg, and restoration of cytosine via follow-on base excision repair [41]. Unlike in physiological de-methylation in germ-line cells an alternate pathway which involves $5 \mathrm{hmC}$ deamination by the Aid/Apobec family of cytidine deaminases before Tdg-mediated base excision repair is not being activated in kidney fibroblasts by hydralazine [41]. Although the de-methylating activity of Hydralazine is inferior to that of 5'-Azacytidine, it is sufficient to re-activate Rasall expression and normalize intrinsic proliferative activity of fibrotic renal fibroblasts - without inducing potentially genotoxic side effects like 5'Aza [40]. Of note, in kidney fibroblasts only Tet3 of the family of Ten-Eleven Translocation enzymes is induced, which does not rule out the possibility that other Tet-family members are involved in other organs [40]. Importantly, hydralazine-induced Tet3 does not cause uncontrolled de-methylation, but targets only select genes - including RASAL1 - which have a CXXC motifs flanking methylated CpG islands $[22,40]$. Such demethylating activity of hydralazine is already being utilized, as phase III clinical trials are ongoing which test impact of hydralazine mediated-demethylation on cancer progression $[42,43]$. Importantly, de-methylation is induced by hydralazine by doses whch are lower than those, which are typically used to lower blood pressure: In clinical cancer trials $3 \times 25 \mathrm{mg} /$ day hydralazine were determined as optimum de-methylating dose, which is in line with the optimum dose of 1-2 times $25 \mathrm{mg}$ /day dihydralazine which were identified in our studies (dihydralazine has a longer half life as compared to hydralazine$\mathrm{HCl}$ ) [42]. In contrast to hydralazine, the established de-methylating drug 5'Aza is incorporated into DNA where it inactivates Dnmts by trapping them through formation of covalent complex formation. In addition, 5'Aza incorporation directly causes DNA damage, which in turn initiates DNA repair mechanisms which also result in replacement with naked unmethylated DNA [44]. However, such DNA damage can also directly initiate cell death [44]. Hence, from an epigenetic standpoint, hydralazine provides sufficient de-methylating activity, combined with a benign side-effect profile.

\section{Hydralazine revisited}

In summary, aberrant DNA methylation of specific genes including RASAL1 - contributes causally to progression of fibrosis in the kidney, as well as in other organs including the heart $[19,22]$. There is evidence that such aberrant fibrotic DNA methylation can be detected in the blood test [40]. Finally, there is evidence that such aberrant DNA methylation can be reversed by treatment with hydralazine, opening the possibility for a biomarker-stratified anti-fibrotic therapy with hydralazine [40]. In this regard, hydralazine (which is primarily used in the U.S., as Apresoline) and its derivate dihydralazine (which is primarily used in Europe as Nepresol) have in in clinical use as anti-hypertensives for more than 60 years, providing substantial evidence for their safety and anti-hypertensive effectiveness [40,45]. Hydralazine is the oldest anti-hypertensive drug which has remained on the market, with primay applications being pregnancy hypertension, hypertension in ICU settings and as third-line add-on anti-hypertensive in complicated hypertension. Due to new novel mechanistic insights, hydralazine may has to be re-visited in comparison to inhibitors of the renin-angiotensinaldosterone system (RAAS) such as angiotensin converting enzyme inhibitors (ACEIs) and angiotensin receptor blockers (ARBs). Today RAAS-inhibitors are the gold-standard in therapy of hypertension, chronic heart failure and proteinuric kidney diseases, because numerous large controlled clinical trials demonstrated efficacy in reducing blood pressure, but also in improving chronic heart failure and in reducing 
cardiovascular mortality and in reducing proteinuria and improving outcome of proteinuric chronic kidney diseases shortly after their introduction to clinical use [40,46-49]. In comparison, hydralazine was introduced to the clinic in 1952, before the age of modern clinical trials, and the first small controlled clinical study on efficacy of hydralazine in hypertension was not reported before 1964 [50]. Nevertheless, hydralazine as part of "standard-triple therapy" was the gold-standard for anti-hypertensive therapy [51,52] - until ACEIs emerged and their superior effectiveness to lower blood pressure and more importantly to reduce cardiovascular mortality at optimum doses was proven $[53,54]$. Hydralazine received renewed interest, when a fixed combination of hydralazine and isosorbit dinitrate (BiDil, marketed as new drug then as patent protection for hydralazine had long run out) showed effectiveness to improve chronic heart failure [55]. While BiDil was effective in all patients, it was only superior to ACEIs (at optimum dose) in self-declared African Americans (but not in Caucasians), and FDA approval was only granted for use in African Americans - making BiDil the first "race-based drug" [56,57]. However, hydralazine in treatment was only inferior to optimally dosed RAAS inhibitors and it may have to be considered that optimum doses of RAAS inhibitors (i.e., for treatment of heart failure $10 \mathrm{mg} /$ day Ramipril or $320 \mathrm{mg} /$ day Valsartan) are often not reached due to decrease of glomerular filtration rate, hyperkalemia or hypotension. In this regard, hydralazine does not require dose reduction it cases of low GFR, making it a possible substitute to RAAs inhibitors, even when its de-methylating efficacy is not even taken into account $[58,59]$. In fact, studies comparing efficacy of ACEIs to hydralazine concluded that combination of ACEIs and hydralazine should be tested - but such trials have not yet been done. To this ongoing discussion the new insights on the impact of epigenetics and de-methylating activity of hydralazine now add an entirely new aspect. When considering possibility of substituting hydralazine for RAAS in clinical scenarios when RAAS inhibitors can not be used at optimal doses, it has to be taken into account that optimal demethylating activity of hydralazine is already achieved at doses which are below blood pressure lowering regimen. In retrospective analysis we documented effective RASAL1 de-methylation and reno-protection over a one-year period in patients with CKD stage 4 with hypertensive nephrosclerosis at dihydralazine doses of $2 \times$ daily $12.5 \mathrm{mg}$. In these patients systolic and diastolic blood pressure was not affected by lowdose dihydralazine, confirming that observed reno-protection was independent of possible anti-hpertensive effects of dihydralazine. These results raised the possibility that dihydralazine could be used in be utilized in biomarker-stratified fashion, even without risk of hypotensive episodes and reflex tachycardia, which can be observed upon standard dose (100-200 mg) dihydralazine. Furthermore, such de-methylating effectiveness of low-dose hydralazine conceptually makes combination of hydralazine and RAAS inhibitors an attractive therapeutic combinations in patients with chronic heart and/ or chronic kidney failure, which may deserve clinical testing after all. Considering the safety of hydralazine, there may be a lot to gain at a very low risk.

\section{Authors' contribution}

$M Z$ wrote the manuscript. EMZ wrote the manuscript.

\section{Acknowledgement}

This work was in part supported by grants from Deutsche Forschungsgemeinschaft ZE523/4-1 to MZ and SFB1002/TPC01 to EZ and funds from the University of Göttingen Medical Center (UMG).

\section{References}

1. Remuzzi G, Bertani T (1998) Pathophysiology of progressive nephropathies. N Engl J Med 339: 1448-1456.
2. Genovese F, Manresa AA, Leeming DJ, Karsdal MA, Boor P (2014) The extracellular matrix in the kidney: a source of novel non-invasive biomarkers of kidney fibrosis? Fibrogenesis Tissue Repair 7: 4

3. Jha V, Garcia-Garcia G, Iseki K, Li Z, Naicker S, et al. (2013) Chronic kidney disease: global dimension and perspectives. Lancet 382: 260-272.

4. Zeisberg M, Zeisberg EM (2015) Precision renal medicine: a roadmap towards targeted kidney fibrosis therapies. Fibrogenesis Tissue Repair 8: 16.

5. Zeisberg M, Neilson EG (2010) Mechanisms of tubulointerstitial fibrosis. J Am Soc Nephrol 21: 1819-1834.

6. Saito S, Tampe B, Müller GA, Zeisberg M (2015) Primary cilia modulate balance of canonical and non-canonical Wnt signaling responses in the injured kidney. Fibrogenesis Tissue Repair 8: 6.

7. Charytan DM, Cinelli A, Zeisberg EM (2015) Association of circulating angiogenesis inhibitors and asymmetric dimethyl arginine with coronary plaque burden. Fibrogenesis Tissue Repair 8: 13

8. Rockey DC, Bell PD, Hill JA (2015) Fibrosis--a common pathway to organ injury and failure. N Engl J Med 372: 1138-1149.

9. Wynn TA (2008) Cellular and molecular mechanisms of fibrosis. J Pathol 214 199-210.

10. Rodemann HP, Muller GA (1991) Characterization of human renal fibroblasts in health and disease: II. In vitro growth, differentiation, and collagen synthesis of fibroblasts from kidneys with interstitial fibrosis. Am J Kidney Dis 17: 684-686.

11. Zeisberg M, Kalluri R (2013) Cellular mechanisms of tissue fibrosis. 1. Common and organ-specific mechanisms associated with tissue fibrosis. Am J Physio Cell Physiol 304: C216-225.

12. Kalluri R, Zeisberg M (2006) Fibroblasts in cancer. Nat Rev Cancer 6: 392-401

13. Orimo A, Gupta PB, Sgroi DC, Arenzana-Seisdedos F, Delaunay T, et al. (2005) Stromal Fibroblasts Present in Invasive Human Breast Carcinomas Promote Tumor Growth and Angiogenesis through Elevated SDF-1/CXCL12 Secretion. Cell 121: 335-348

14. Zeisberg EM, Zeisberg M (2013) The role of promoter hypermethylation in fibroblast activation and fibrogenesis. J Pathol 229: 264-273.

15. Susztak K (2014) Understanding the epigenetic syntax for the genetic alphabet in the kidney. J Am Soc Nephrol 25: 10-17.

16. Wing MR, Devaney JM, Joffe MM, Xie D, Feldman HI, et al. (2014) DNA methylation profile associated with rapid decline in kidney function: findings from the CRIC study. Nephrol Dial Transplant 29: 864-872.

17. Tampe D, Zeisberg M (2012) A primer on the epigenetics of kidney fibrosis Minerva Med 103: 267-278.

18. Herman JG, Baylin SB (2003) Gene silencing in cancer in association with promoter hypermethylation. N Engl J Med 349: 2042-2054.

19. Bechtel W, McGoohan S, Zeisberg EM, Müller GA, Kalbacher H, et al. (2010) Methylation determines fibroblast activation and fibrogenesis in the kidney. Nat Med 16: 544-550.

20. Zeybel M, Hardy T, Wong YK, Mathers JC, Fox CR, et al. (2012) Multigenerationa epigenetic adaptation of the hepatic wound-healing response. Nat Med 18 1369-1377.

21. Xu X, Friehs I, Hu TZ, Melnychenko I, Tampe B, et al. (2015) Endocardial fibroelastosis is caused by aberrant endothelial to mesenchymal transition. Circ Res 116: 857-866.

22. Xu X, Tan X, Tampe B, Nyamsuren G, Liu X, et al. (2015) Epigenetic balance of aberrant Rasal1 promoter methylation and hydroxymethylation regulates cardiac fibrosis. Cardiovasc Res 105: 279-291.

23. Dees C, Schlottmann I, Funke R, Distler A, Palumbo-Zerr K, et al. (2014) The Wnt antagonists DKK1 and SFRP1 are downregulated by promoter hypermethylation in systemic sclerosis. Ann Rheum Dis 73: 1232-1239.

24. Baylin SB, Ohm JE (2006) Epigenetic gene silencing in cancer - a mechanism for early oncogenic pathway addiction? Nat Rev Cancer 6: 107-116.

25. De Los Angeles A, Ferrari F, Xi R, et al. (2015) Hallmarks of pluripotency. Nature 525: 469-478.

26. Rhee I, Jair KW, Yen RW, Lengauer C, Herman JG, et al. (2000) CpG 
Citation: Zeisberg EM, Zeisberg M (2016) Inhibition of Fibrogenesis upon Hydralazine-induced DNA Demethylation. J Kidney 2: 124. doi:10.4172/24721220.1000124

Page 5 of 5

methylation is maintained in human cancer cells lacking DNMT1. Nature 404: 1003-1007.

27. Rhee I, Bachman KE, Park BH, Jair KW, Yen RW, et al. (2002) DNMT1 and DNMT3b cooperate to silence genes in human cancer cells. Nature 416: 552-556.

28. Neary R, Watson CJ, Baugh JA (2015) Epigenetics and the overhealing wound: the role of DNA methylation in fibrosis. Fibrogenesis Tissue Repair 8: 18

29. Sun CY, Chang SC, Wu MS (2012) Suppression of Klotho expression by proteinbound uremic toxins is associated with increased DNA methyltransferase expression and DNA hypermethylation. Kidney Int 81: 640-650.

30. Downward J (2003) Targeting RAS signalling pathways in cancer therapy. Nat Rev Cancer 3: 11-22.

31. Mitin N, Rossman KL, Der CJ (2005) Signaling interplay in Ras superfamily function. Curr Biol 15: R563-574.

32. Sharpe CC, Dockrell ME, Noor MI, Monia BP, Hendry BM (2000) Role of Ras isoforms in the stimulated proliferation of human renal fibroblasts in primary culture. J Am Soc Nephrol 11: 1600-1606.

33. Smaldone S, Olivieri J, Gusella GL, Moroncini G, Gabrielli A, et al. (2011) Ha-Ras stabilization mediates pro-fibrotic signals in dermal fibroblasts. Fibrogenesis Tissue Repair 4: 8.

34. Hendry BM, Sharpe CC (2003) Targeting Ras genes in kidney disease. Nephron Exp Nephrol 93: e129-133.

35. Cullen PJ, Lockyer PJ (2002) Integration of calcium and Ras signalling. Nat Rev Mol Cell Biol 3: 339-348.

36. Reuther GW, Der CJ (2000) The Ras branch of small GTPases: Ras family members don't fall far from the tree. Curr Opin Cell Biol 12: 157-165.

37. Walker SA, Kupzig S, Bouyoucef D, Davies LC, Tsuboi T, et al. (2004) Identification of a Ras GTPase-activating protein regulated by receptormediated Ca2+ oscillations. EMBO J 23: 1749-1760.

38. Kolfschoten IG, van Leeuwen B, Berns K, Mullenders J, Beijersbergen RL, et al. (2005) A genetic screen identifies PITX1 as a suppressor of RAS activity and tumorigenicity. Cell 121: 849-858.

39. Tampe B, Tampe D, Muller C, Sugimoto H, LeBleu V, et al. (2014) Tet3 mediated hydroxymethylation of epigenetically silenced genes contributes to BMP7-induced Reversal of Kidney Fibrosis. J Am Soc Nephrol.

40. Tampe B, Tampe D, Zeisberg EM, Müller GA, Bechtel-Walz W, et al. (2015) Induction of Tet3-dependent Epigenetic Remodeling by Low-dose Hydralazine Attenuates Progression of Chronic Kidney Disease. EBioMedicine 2: 19-36.

41. Bhutani N, Burns DM, Blau HM (2011) DNA demethylation dynamics. Cell 146: 866-872.

42. Coronel J, Cetina L, Pacheco I, Trejo-Becerril C, Gonzalez-Fierro A, et al. (2011) A double-blind, placebo-controlled, randomized phase III trial of chemotherapy plus epigenetic therapy with hydralazine valproate for advanced cervical cancer. Preliminary results. Med Oncol 28: S540-S546.

43. Zambrano P, Segura-Pacheco B, Perez-Cardenas E, Cetina L, Revilla-Vazquez A, et al. (2005) A phase I study of hydralazine to demethylate and reactivate the expression of tumor suppressor genes. BMC Cancer 5: 44

44. Buckstein R, Yee K, Wells RA (2011) 5-Azacytidine in myelodysplastic syndromes: a clinical practice guideline. Cancer Treat Rev 37: 160-167.
45. Krimsky S (2012) The short life of a race drug. Lancet 379: 114-115.

46. Murphy AM, Wong AL, Bezuhly M (2015) Modulation of angiotensin II signaling in the prevention of fibrosis. Fibrogenesis Tissue Repair 8: 7.

47. van der Meer IM, Cravedi P, Remuzzi G (2010) The role of renin angiotensin system inhibition in kidney repair. Fibrogenesis Tissue Repair 3: 7.

48. Anderson S, Rennke HG, Brenner BM (1986) Therapeutic advantage of converting enzyme inhibitors in arresting progressive renal disease associated with systemic hypertension in the rat. J Clin Invest 77: 1993-2000.

49. Rauen T, Eitner F, Fitzner C, Sommerer C, Zeier M, et al. (2015) Intensive Supportive Care plus Immunosuppression in IgA Nephropathy. N Engl J Med 373: $2225-2236$

50. Smith WM, Damato AN, Galluzzi NJ, Garfield CF, Hanowell EG, et al. (1964) The Evaluation of Antihypertensive Therapy Cooperative Clinical Trial Method. I. Double-Blind Control Comparison of Chlorothiazide, Rauwolfia Serpentina, and Hydralazine. Ann Intern Med 61: 829-846.

51. Andersson O, Hansson L, Sivertsson R (1978) Primary hypertension refractory to triple drug treatment: a study on central and peripheral hemodynamics. Circulation 58: 615-622.

52. Vandenburg MJ, Wright P, Holmes J, Rogers HJ, Ahmad RA (1982) The hypotensive response to hydralazine, in triple therapy, is not related to acetylator phenotype. Br J Clin Pharmacol 13: 747-750.

53. The CONSENSUS Trial Study Group (1987) Effects of enalapril on mortality in severe congestive heart failure. Results of the Cooperative North Scandinavian Enalapril Survival Study (CONSENSUS). N Engl J Med 316:1429-1435.

54. The SOLVD Investigator (1991) Effect of enalapril on survival in patients with reduced left ventricular ejection fractions and congestive heart failure. $\mathrm{N}$ Engl J Med 325: 293-302.

55. Farag M, Mabote T, Shoaib A, Zhang J, Nabhan AF, et al. (2015) Hydralazine and nitrates alone or combined for the management of chronic heart failure: $A$ systematic review. Int J Cardiol 196: 61-69.

56. Dries DL, Exner DV, Gersh BJ, Cooper HA, Carson PE, et al. (1999) Racia differences in the outcome of left ventricular dysfunction. N Engl J Med 340 609-616.

57. Taylor AL, Ziesche S, Yancy C, Carson P, D’Agostino R Jr, et al. (2004) Combination of isosorbide dinitrate and hydralazine in blacks with heart failure. N Engl J Med 351: 2049-2057.

58. Cohn JN, Tognoni G (2001) Valsartan Heart Failure Trial Investigators A randomized trial of the angiotensin-receptor blocker valsartan in chronic heart failure. N Engl J Med 345: 1667-1675.

59. Hall AS, Murray GD, Ball SG (1997) Follow-up study of patients randomly allocated ramipril or placebo for heart failure after acute myocardial infarction: AIRE Extension (AIREX) Study. Acute Infarction Ramipril Efficacy. Lancet 349 $1493-1497$. 